\title{
Origin of density clustering in a freely evolving granular gas
}

\author{
J. Javier Brey, M. J. Ruiz-Montero, and D. Cubero \\ Física Teórica, Universidad de Sevilla, Apartado de Correos 1065, E-41080 Sevilla, Spain
}

(Received 14 January 1999; revised manuscript received 12 May 1999)

\begin{abstract}
The physical mechanisms leading to the development of density inhomogeneities in a freely evolving low density granular gas are investigated. By means of the direct simulation Monte Carlo method, numerical solutions of the inelastic Boltzmann equation are constructed for both a perturbed system and also for an initially homogeneous state. Analysis of the Fourier components of the fields indicates that the nonlinear coupling contributions of the transversal velocity play a crucial role in the initial setup of clustering. A simple hydrodynamic model is proposed to describe what is observed in the simulations. Finally, the nature of the inhomogeneous state is briefly discussed. [S1063-651X(99)08909-6]
\end{abstract}

PACS number(s): 81.05.Rm, 45.05.+x, 05.20.Dd, 47.20.-k

\section{INTRODUCTION}

One of the characteristic features of granular gases, as compared with molecular ones, is their tendency to form density clusters when evolving freely [1-3]. This seems to be a hydrodynamic instability that follows from the presence of a dissipation term in the equation for the balance of energy. Goldhirsch and Zanetti [1] proposed the following mechanism to explain the cluster formation. Suppose that at a given moment the density in a region of the fluid increases due to a localized fluctuation. Then the collision frequency also increases, and the temperature drops faster than in the surrounding fluid, due to the energy dissipation in collisions. If, as a consequence, the pressure in that region falls below the average value, more particles are attracted and a density cluster begins to develop.

Although the above heuristic argument seems very clear, translating it into a more quantitative theory is not easy at all. In fact, two different origins have been proposed in the literature as responsible for the initial density nonuniformity from which the clustering process proceeds. In Ref. [1], a fluctuation of the shear mode was considered. After a transient time, nonlinear hydrodynamic effects dominate the dynamics of the system and, in particular, the evolution equation for the granular temperature, that grows until reaching a saturation value. Then the pressure balance is violated and, from the equation of state, it follows that the lower the pressure in a region the higher the density. Another different possibility is that the initial density fluctuation is just a linear effect associated with a fluctuation of the mode governing the linear evolution of the longitudinal component of the flow field $[4,5]$. The main aim of this paper is to discriminate between the two above mechanisms by using the direct simulation Monte Carlo method to obtain numerical solutions of the Boltzmann equation. This allows us to follow in detail the temporal and spatial properties of the hydrodynamic fields, and to compare them with the predictions following from each of the two theories. In this sense, we notice that the numerical results we will present here do not assume the validity of a hydrodynamic description for a granular gas, although they rest on the accuracy of the Boltzmann equation to describe a dilute gas of smooth inelastic hard spheres.

To carry out the comparison of the simulation data with the nonlinear hydrodynamic theory, it is necessary to develop some quantitative consequences of the latter. Analytical results for the nonlinear behavior of granular flows are rare. Here we propose a very simple model that is specially designed to describe the numerical experiments we have carried out. The main ingredient is the fact that contributions to the shear mode of large wavelength grow in time, while those corresponding to short wavelengths decay. The concepts of large and short are related in this context to the inelasticity of collisions.

The paper is organized as follows. In Sec. II we describe the basis of the continuous hydrodynamic equation for a low density granular gas as derived from the (inelastic) Boltzmann equation. Moreover, the results from the linear stability analysis about the homogeneous cooling state are shortly reviewed. In Sec. III the numerical results obtained for a system whose macroscopic velocity field is initially perturbed are presented. A simple theoretical model is proposed, and its predictions compared with the simulation results in Sec. IV. The case of a spontaneous fluctuation is also discussed. Finally, in Sec. V some further comments are addressed. In particular, the nature of the inhomogeneous state reached for the freely evolving granular gas as a consequence of the instability is discussed.

\section{HYDRODYNAMIC DESCRIPTION}

The hydrodynamic description of a rapid granular flow is made in terms of the same fields as an ordinary fluid, namely, the local number density $n(\mathbf{r}, t)$, the velocity field $\mathbf{u}(\mathbf{r}, t)$, and the local temperature $T(\mathbf{r}, t)[6]$. To second order in the gradients, the time evolution of these fields for a low density gas of smooth inelastic hard spheres of diameter $\sigma$ and mass $m$ is given by [7]

$$
\begin{gathered}
\partial_{t} n+\nabla \cdot(n \mathbf{u})=0, \\
\partial_{t} \mathbf{u}+\mathbf{u} \cdot \nabla \mathbf{u}+(n m)^{-1} \nabla \cdot \mathrm{P}=0, \\
\partial_{t} T+\mathbf{u} \cdot \nabla T+2\left(3 n k_{B}\right)^{-1}(\mathrm{P}: \nabla \mathbf{u}+\nabla \cdot \mathbf{q})+T\left(\zeta^{(0)}+\zeta^{(2)}\right)=0,
\end{gathered}
$$

where $k_{B}$ is the Boltzmann constant, $P_{i j}=p \delta_{i j}-\eta\left(\nabla_{i} u_{j}\right.$ $\left.+\nabla_{j} u_{i}-2 \delta_{i j} \nabla \cdot \mathbf{u} / 3\right)$ the pressure tensor, and $\mathbf{q}=-\kappa \nabla T$ 
$-\mu \nabla n$ the heat flux. Here $p=n k_{B} T$ is the hydrodynamic pressure, $\eta$ the shear viscosity, $\kappa$ the heat conductivity, and $\mu$ a transport coefficient which has no elastic analog. The loss of energy in collisions is accounted for through $\zeta^{(0)}$ and $\zeta^{(2)}$. While the former is a single term of zeroth order in the gradients, the latter is given by a sum of second order in the gradients contributions:

$$
\begin{aligned}
\zeta^{(2)}= & \zeta_{1} \nabla^{2} T+\zeta_{2} \nabla^{2} n+\zeta_{3}(\nabla T)^{2}+\zeta_{4}(\nabla n)^{2}+\zeta_{5} \nabla T \cdot \nabla n \\
& +\zeta_{6} \nabla \mathbf{u}: \nabla \mathbf{u}+\zeta_{7} \nabla \mathbf{u}:(\nabla \mathbf{u})^{+} .
\end{aligned}
$$

The expressions for the transport coefficients are of the forms

$$
\eta=\eta^{*}(\alpha) \eta_{0}, \quad \kappa=\kappa^{*}(\alpha) \kappa_{0}, \quad \mu=\mu^{*}(\alpha) \frac{\kappa_{0} T}{n},
$$

with $\eta_{0}=5\left(m k_{B} T\right)^{1 / 2} / 16 \sigma^{2} \pi^{1 / 2}$ and $\kappa_{0}=15 k_{B} \eta_{0} / 4 m$ being the elastic limit values of the shear viscosity and thermal conductivity, respectively. The quantities $\eta^{*}, \kappa^{*}$, and $\mu^{*}$ are dimensionless functions of the coefficient of normal restitution $\alpha$. In a similar way, it is

$$
\zeta^{(0)}=\zeta^{*}(\alpha) \frac{n k_{B} T}{\eta_{0}}
$$

and $\zeta^{*}(\alpha)$ vanishes in the elastic limit. The explicit expressions of $\eta^{*}, \kappa^{*}, \mu^{*}$, and $\zeta^{*}$ are given in the Appendix, and more details can be found in Ref. [7]. Also in the Appendix, the coefficients $\zeta_{1}$ and $\zeta_{2}$ of the linear terms in Eq. (4) are given. For not very inelastic particles, both linear terms can be accurately neglected in the transport equations, although they have been kept in the results we will report in the following.

The hydrodynamic equations admit a simple solution describing a uniform system with vanishing flow field and a temperature decreasing monotonically in time according to the law

$$
T_{H}(t)=T_{H}(0)\left(1+t / t_{0}\right)^{-2},
$$

with $t_{0}^{-1}=\zeta^{(0)}(0) / 2$. Molecular dynamics simulations [1-3] and also Monte Carlo simulations of the Boltzmann equation [8] have shown that this homogeneous cooling state (HCS) is unstable and develops high density clusters spontaneously. Our aim here is to investigate which are the leading mechanisms responsible for this instability by means of the hydrodynamic description provided by Eqs. (1)-(3). Linearization of these equations about the hydrodynamic fields defining the HCS leads to equations with coefficients that do not depend on position but are time dependent. In order to eliminate this time dependence, it is convenient to introduce new time and space scales by $d \tau=\nu_{H}(t) d t / 2$ and $d \mathbf{l}$ $=\nu_{H}(t) \mathrm{v}_{H}^{-1}(t) d \mathbf{r} / 2$, respectively, where $\nu_{H}=p_{H} / \eta_{0, H}$ and $\mathrm{v}_{H}=\left(k_{B} T_{H} / m\right)^{1 / 2}$. We also define dimensionless fields as $\rho$ $=\delta n / n_{H}, \boldsymbol{\omega}=\delta \mathbf{u} / \mathrm{v}_{H}$, and $\theta=\delta T / T_{H}$, where $\delta y$ denotes the deviation of the local field $y(\mathbf{r}, t)$ from its value in the reference HCS, $y_{H}$. Now the equations for the Fourier hydrodynamic modes become ordinary differential equations with time independent coefficients. The vector component of

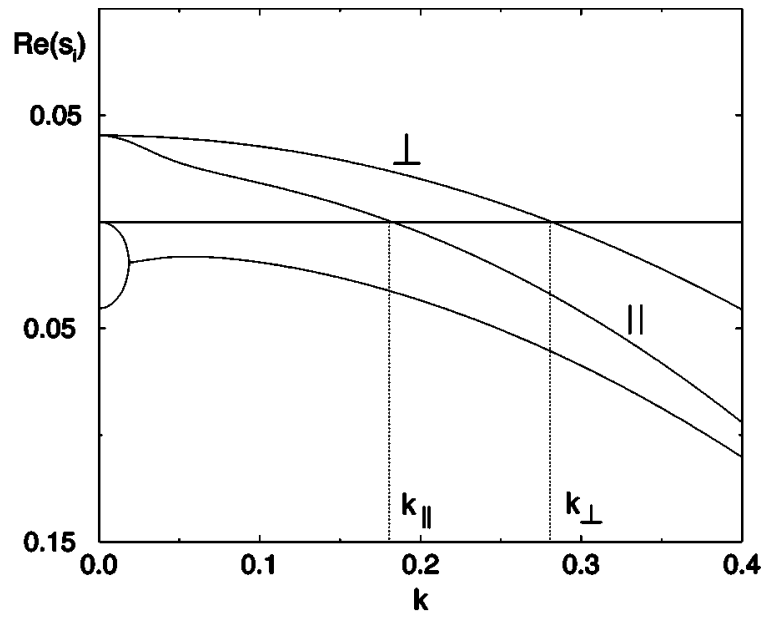

FIG. 1. Dispersion relations for a dilute granular gas with $\alpha$ $=0.95$. Both $s_{\gamma}$ and $\mathbf{k}$ are measured in the dimensionless reduced units defined in the text. Only the real part of the eigenvalues is plotted.

the velocity field perpendicular to the wave vector $\mathbf{k}, \boldsymbol{\omega}_{\mathbf{k} \perp}$, is decoupled from the other hydrodynamic fields, and obeys the equation

$$
\boldsymbol{\omega}_{\mathbf{k} \perp}(\tau)=\omega_{\mathbf{k} \perp}(0) e^{s_{\perp}} \tau,
$$

with $s_{\perp}=\zeta^{*}-\frac{1}{2} \eta^{*} k^{2}$. This identifies two shear modes analogous to the elastic ones. The equations for the density $\rho_{\mathbf{k}}$, the longitudinal component of the velocity $\omega_{\mathbf{k} \|}$, and the temperature $\theta_{\mathbf{k}}$ are coupled, and the solutions have the form of a linear combination of three hydrodynamic modes,

$$
\delta y_{\mathbf{k} \beta}=\sum_{\gamma=1}^{3} c_{\beta \gamma} \varphi_{\gamma}(\alpha, \mathbf{k}) e^{s_{\gamma}(\alpha, k) \tau}
$$

where $\varphi_{\gamma}$ and $s_{\gamma}$ are, respectively, the eigenfunctions and eigenvalues of the eigenproblem associated with the linear system of hydrodynamic equations. The frequencies $s_{\gamma}$ are given by the solutions of a cubic algebraic equation that can be solved numerically. The dispersion relations $s_{\beta}=s_{\beta}(k)$ for a system with $\alpha=0.95$ are shown in Fig. 1. Only the real part of the propagating modes has been plotted. When interpreting the results, it must be noted that, except for the shear modes $(\perp)$, the physical meaning of the modes is different from that in elastic fluids even close to the elastic limit.

Three different regions are identified from the dispersion relations. For $k>k_{\perp}$, where

$$
k_{\perp}=\left(\frac{2 \zeta^{*}}{\eta^{*}}\right)^{1 / 2},
$$

all modes are negative and the system is linearly stable for initial perturbations with wave number in this range (short wavelength region). For $k_{\|}<k<k_{\perp}$, where

$$
k_{\|}=\left[\frac{4 \zeta^{*}}{5\left(\kappa^{*}-\zeta_{1}^{*}-\mu^{*}+\zeta_{2}^{*}\right)}\right]^{1 / 2},
$$


the shear modes are positive, while the real part of the remaining ones are negative. Therefore, initial perturbations of the HCS that excite the shear modes with $k<k_{\perp}$ will grow exponentially with $\tau$. Of course, this refers to the scaled field $\boldsymbol{\omega}_{\mathbf{k} \perp}(\tau)$, which will not be linearly stable.

As a consequence, the system is expected to exhibit vortices whose intensity grows in time when the scaled velocity is considered. This has been confirmed by the simulation results $[1-3,8]$. As the perturbation becomes larger, the full nonlinear hydrodynamic equations are, in principle, required to study the posterior evolution of the system. Nevertheless, this does not mean by itself that the system is hydrodynamically unstable. In fact, taking into account that $T_{H}(\tau)$ $=T_{H}(0) e^{-2 \zeta^{*} \tau}$ and that $s_{\perp}$ is upper bounded by $\zeta^{*}$, it is easily realized that the perturbation of the transversal component of the velocity field $\delta \mathbf{u}_{\mathbf{k} \perp}$ decays monotonically in the linear approximation.

Finally, for $k<k_{\|}$, the parallel mode $(\|)$also has a positive eigenvalue $s_{\|}$, and the excitation grows in time. In general, this mode may contribute to $\omega_{\mathbf{k} \|}, \rho_{\mathbf{k}}$, and $\theta_{\mathbf{k}}$, that would increase exponentially with $\tau$, rendering the linear equations invalid after an initial transient period. The same kind of analysis as above indicates that $\delta u_{\mathbf{k} \|}$ and $\delta T_{\mathbf{k}}$ always decay for large times in the linear approximation, but $\delta n_{\mathbf{k}}$ increases if the initial perturbations excite the parallel mode. As a consequence, the density is linearly unstable for perturbations of this mode with $k<k_{\|}$. It must be realized that this does not imply by itself that the density is asymptotically unstable.

Thus the linear analysis yields two potential mechanisms for the formation of density clusters, both of which have been discussed in the literature. Goldhirsch and Zanetti [1] considered that the dominant effect leading to the development of density inhomogeneities is the nonlinear contribution of the shear mode to the evolution equation for the temperature. The other possibility is that the cluster formation is directly related with the initial exponential growth of a density fluctuation which excites the parallel mode $[4,5]$. Both mechanisms are quite different. While in the former density clusters are closely related with the formation of velocity vortices, in the latter vortices and clusters are independent effects, at least in the first stages of the density buildup. Previous numerical simulations of the inelastic Boltzmann equation have provided indications that the actual scenario is closer to the first possibility mentioned above [9]. The velocity and density fields show similar spatial structures, with the higher density regions corresponding to the borders of the vortices. Here a more detailed and quantitative description will be given.

\section{RESPONSE TO A PERTURBATION OF THE VELOCITY FIELD}

Numerical solutions of the (inelastic) Boltzmann equation can be obtained by means of the direct simulation Monte Carlo (DSMC) method [10]. We have considered a gas of inelastic smooth hard spheres between two planes perpendicular to the $x$ axis separated a distance $L$. Periodic boundary conditions were applied in this direction, while the system was assumed to be homogeneous at all times in the other two directions. Therefore, no boundary conditions were needed along them. In other words, we simulated the equation

$$
\left(\frac{\partial}{\partial t}+\mathbf{v}_{x} \frac{\partial}{\partial x}\right) f=J[f, f]
$$

where $f(x, \mathbf{v}, t)$ is the one-particle distribution function for the gas, that is assumed to be independent of $y$ and $z$, and $J$ is the nonlinear inelastic Boltzmann collision operator [11,7]. It follows that the $y$ and $z$ coordinates of the simulated particles play no role in the simulations, although the three components of the velocity are, of course, relevant in the collision processes.

The consideration of Eq. (12) instead of the most general case, is a very convenient choice to increase the statistics in the relevant direction $x$, in which the spatial perturbations are studied. Of course, we will restrict ourselves in this way to gradients in only this direction. In any case, we want to mention that we have also simulated systems in which gradients along two directions were allowed and no relevant coupling between orthogonal components of the gradients were found. The simulations we will report typically involved $5 \times 10^{6}$ particles, and the system was divided into 120 space cells parallel to the plates. Starting from an homogeneous initial state and an isotropic velocity distribution, the system was allowed to evolve freely for a short period of time, until the HCS was reached. Then, an external perturbation of the flow velocity was introduced by adding to the scaled velocities of all particles a contribution

$$
\boldsymbol{\omega}(x, 0)=\boldsymbol{\omega}_{0} \sin \left(q_{0} x\right),
$$

where $q_{0}=2 \pi / L$ and $\boldsymbol{\omega}_{0}$ is a vector of components $\omega_{0 x}$ $=\omega_{0 y}=\omega_{0 z}=\omega_{0}$. The above expression is compatible with the periodic boundary conditions along the $x$ axis. Notice that in this case, $\omega_{y}$ and $\omega_{z}$ are the transversal components of the velocity, while $\omega_{x}$ is the longitudinal one, since only gradients in the $x$ direction are possible.

The idea behind the above perturbation is to excite the two hydrodynamic modes which are involved, in principle, in the clustering instability. According to the linear theory, in the long wavelength limit, a fluctuation of the longitudinal component of the flow field induces a fluctuation of the density that after an initial transient time grows exponentially with $\tau$ at a rate given by $s_{\|}[5,13]$. On the other hand, fluctuations of the transversal components of the flow field, although evolving separately in the linear approximation, can couple to the other fields in the nonlinear regime, leading to a growth of the density inhomogeneities.

Once the perturbation in Eq. (13) is introduced, there is a time interval for which the macroscopic dynamics of the system is well described by the linear hydrodynamic equations [12]. Afterwards, nonlinear effects begin to show up. In order to discriminate between linear and nonlinear effects, it is convenient to choose the size of the system in an appropriate way. Because of the periodic boundary conditions, the only allowed values of the $x$ component of the wave vector in a Fourier analysis are multiples of $q_{0}$ or, in the reduced units we are employing, multiples of $k_{0}=2 \mathrm{v}_{H} \nu_{H}^{-1} q_{0}$. Suppose we take $L$ such that the transversal velocity mode with $k=k_{0}$ is linearly unstable, but the one with $k=2 k_{0}$ is stable. It is clear 

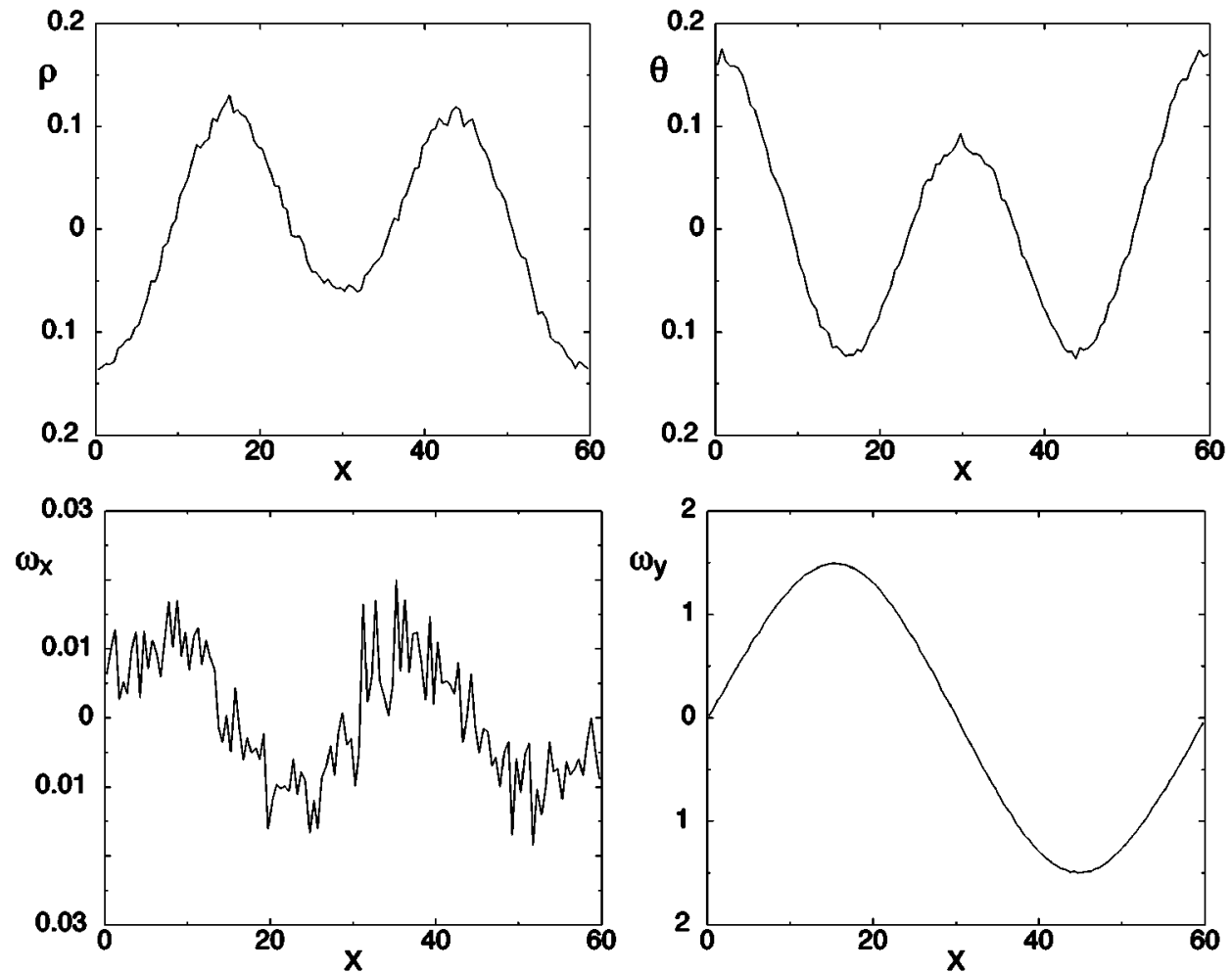

FIG. 2. Density $(\rho)$, temperature $(\theta)$, longitudinal velocity $\left(\omega_{x}\right)$, and transversal velocity $\left(\omega_{y}\right)$ profiles at a time $\tau=148.5$ following a harmonic perturbation of the velocity field given by Eq. (13). All fields are measured in the reduced units defined in the text. The distance $x$ is measured in units of the mean free path $\lambda$. The coefficient of restitution is $\alpha$ $=0.95$.

that the appearance and growth of a component of any (scaled) hydrodynamic field corresponding to the mode $2 k_{0}$ can be due only to nonlinear hydrodynamic couplings.

In Fig. 2 we report numerical results for the hydrodynamic profiles obtained in a system with $\alpha=0.95$ and $L$ $=60 \lambda$, where $\lambda=1 / \sqrt{2} n \pi \sigma^{2}$ is the mean free path. For this system $k_{0} \simeq 0.164$, and from the dispersion relations in Fig. 1 it follows that $k_{0}<k_{\|}<k_{\perp}$ but $2 k_{0}>k_{\perp}$. The initial amplitude of the perturbation was $\omega_{0}=0.05 \sqrt{2}$. Let us point out that there is a compromise in the choice of $k_{0}$ or, equivalently, of the size $L$. We wanted $k_{0}$ to be close enough to $k_{\|}$ so that the mode with $2 k_{0}$ were linearly stable, but at the same time it should be small enough so that the hydrodynamic description given by Eqs. (1)-(3), which is restricted to small gradients, applied. The value $L=60 \lambda$ for $\alpha=0.95$ turned out to fulfill both requirements.

The profiles plotted in Fig. 2 correspond to a time $\tau$ $=148.5$. It is seen that, for this time, the transversal component of the velocity $\omega_{y}$ is still well described by the sinus function associated to the lowest harmonic. Even more, the analysis of the time evolution of its amplitude shows that it is in good agreement with the prediction of the linear law given by Eq. (8) [12]. A similar behavior is found for $\omega_{z}(\tau)$. On the other hand, a simple glance at the figure indicates that the density, temperature, and longitudinal velocity profiles are dominated by the second harmonic, i.e., the Fourier component corresponding to $k=2 k_{0}$. In addition, the numerical data show that the amplitudes of these profiles are increasing in time, contrary to the results obtained from the linear analysis of the hydrodynamic equations. Nevertheless, note that the amplitude of the longitudinal velocity perturbation, $\omega_{x}$, remains bounded by its initial value, while the amplitude of the perturbation of the transversal components has increased two orders of magnitude. All this suggests quite strongly that the transversal velocity field has enslaved the other hydrodynamic fields through some nonlinear coupling of the hydrodynamic modes. Similar results have been obtained for other values of $\alpha$ in the range $0.675 \leqslant \alpha \leqslant 0.95$.

\section{NONLINEAR HYDRODYNAMIC MODE CONTRIBUTIONS}

Since the main contribution to the nonlinear behavior of the system appears to be due, at least in the first stages of the evolution, to the transversal components of the flow velocity, we have tried to build up a hydrodynamic theory based on this idea. For a fluctuation $\delta \mathbf{u}(x)$, the nonlinear viscous heating term due to the shear flow in the equation for the temperature is $-2\left(3 n k_{B}\right)^{-1} \eta\left[\left(\partial \delta u_{y} / \partial x\right)^{2}+\left(\partial \delta u_{z} / \partial x\right)^{2}\right]$. If this term is kept when linearizing the equation for the temperature about the HCS, we obtain

$$
\begin{aligned}
{\left[\frac{\partial}{\partial \tau}+\zeta^{*}+\frac{5}{4}\left(\kappa^{*}-\zeta_{1}^{*}\right) k^{2}\right] \theta_{k}+\left[2 \zeta^{*}+\frac{5}{4}\left(\mu^{*}-\zeta_{2}^{*}\right) k^{2}\right] \rho_{k} } \\
+\frac{2}{3} i k \omega_{x k}+\frac{1}{3} \eta^{*} \sum_{j_{1}=-\infty}^{\infty} j_{1} k_{0}^{2}\left(j-j_{1}\right) \\
\quad \times\left[\omega_{y\left|j_{1} k_{0}\right|} \omega_{y\left|\left(j-j_{1}\right) k_{0}\right|}+\omega_{z\left|j_{1} k_{0}\right|} \omega_{z\left|\left(j-j_{1}\right) k_{0}\right|}\right]=0
\end{aligned}
$$

where we have written $k=j k_{0}$, with $j$ an integer. We have taken into account that only gradients in the $x$ direction are considered in our simulation. The Fourier transforms of the hydrodynamic fields have been defined as

$$
\theta_{k}=\frac{1}{L} \int_{0}^{L} d x \theta(x) e^{-i k x}
$$




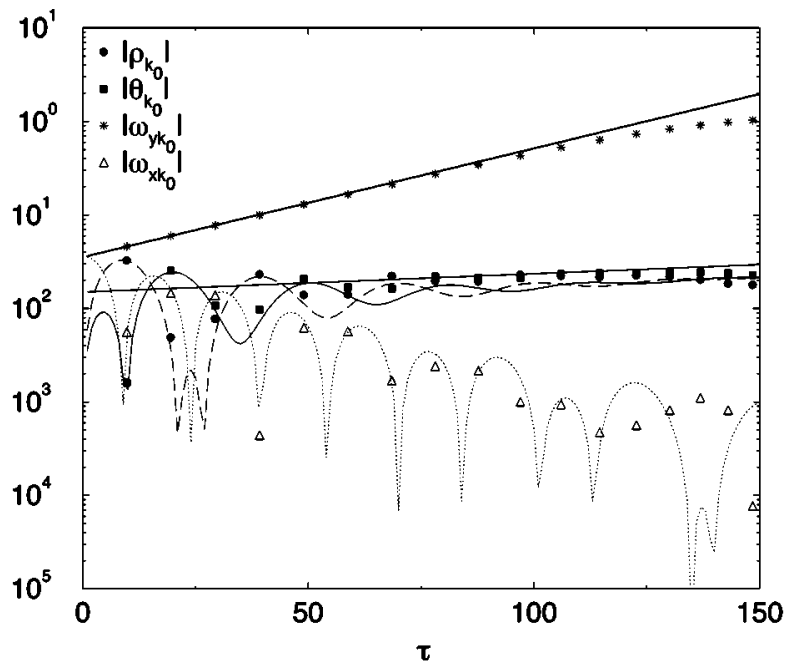

FIG. 3. Time evolution of the lowest Fourier components of the hydrodynamic fields following an harmonic isotropic velocity perturbation given by Eq. (13). The fields and the time $\tau$ are measured in the dimensionless units defined in the text. The symbols are from the simulation and the lines the theoretical predictions from Eq. (14).

and similarly for $\rho$ and $\boldsymbol{\omega}$.

One more approximation will be introduced in order to render the system of hydrodynamic equations simpler. We have already discussed that the transversal component of the velocity field is still well described by the linear approximation when the other fields show already strong nonlinear influences. Then, we will substitute $\omega_{y k}(\tau)$ and $\omega_{z k}(\tau)$ in Eq. (14) by $\omega_{0} \exp \left(s_{\perp} \tau\right)$, i.e., by their expressions in the linear approximation [Eq. (8)]. In this way, Eq. (14) becomes a linear although inhomogeneous ordinary differential equation, to be considered together with the linearization of Eqs. (1) and (2) [7].

The above approximations have been prompted by the numerical results shown in Fig. 1, and indicating the relevant role played by the transversal velocity field. Of course, we do not expect them to hold in order to describe the complete development of the instability, but only the early nonlinear stages. In any case, the ultimate justification will be provided by the comparison of the model implications with the numerical results obtained from the Boltzmann equation. This will be done below.

Let us analyze the implications of Eq. (14). For $k=k_{0}$, i.e., $j=1$, one or both of the numbers $j_{1}$ and $j-j_{1}$ appearing in the last term of the right hand side is equal or larger than 2 . For $k \geqslant 2 k_{0}$ it is $s_{\perp}(k)<0$, because of the system size we are using. As a consequence, the whole last term on the left side of Eq. (14) can be neglected for $k=k_{0}$. That means that the lowest Fourier mode is accurately described by the linear approximation even when the system is well inside the nonlinear region. According with the model we have proposed, this holds for all the hydrodynamic fields. To check this prediction we have carried out a Fourier analysis of the hydrodynamic profiles in the same system as in Fig. 2. The results for $k=k_{0}$ are compared with the solution of the linearized hydrodynamic equations in Fig. 3. This solution has been obtained by solving numerically the linearized equations with the initial conditions $\rho_{k}(0)=0, \theta_{k}(0)=0$, and $\boldsymbol{\omega}_{k}(0)$

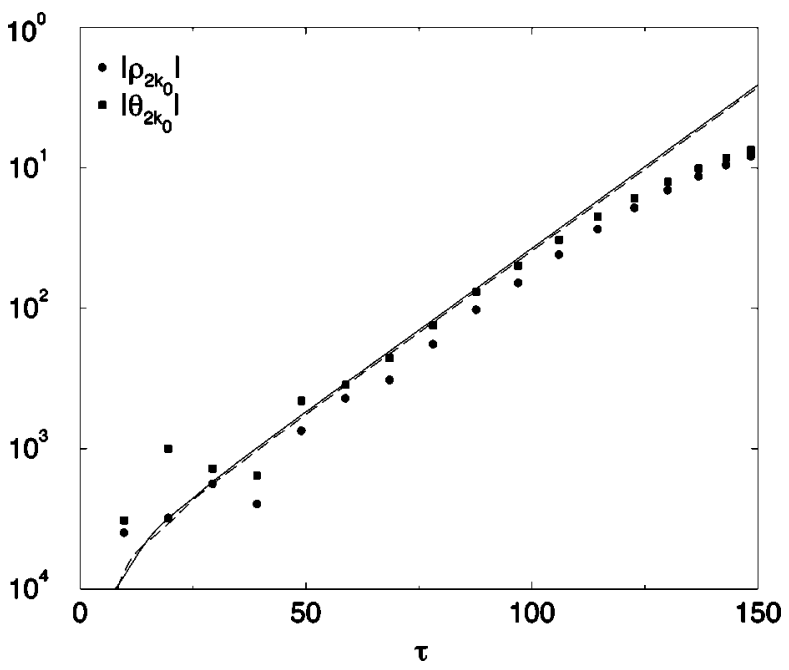

FIG. 4. The same as in Fig. 3 but for the second harmonic, i.e., for $k=2 k_{0}$.

given by the Fourier transform of Eq. (13). There is a quite good agreement between the solution of the Boltzmann equation obtained by the DSMC method and the linearized hydrodynamic description. The level of noise for the longitudinal component of the velocity is very high since the signal is very low. Let us remark that this agreement is a nontrivial result, since the analysis extends well inside the nonlinear region, as it follows from the shape of the profiles for $\tau=148.5$ reported in Fig. 2 .

Next we considered the Fourier components of the hydrodynamic fields with $k=2 k_{0}$. In this case, the term corresponding to $j=1$ in the sum on the right side of Eq. (14) gives an increasing in time contribution. Therefore, the dominant part of the equation reads

$$
\begin{aligned}
{\left[\frac{\partial}{\partial \tau}+\zeta^{*}+\frac{5}{4}\left(\kappa^{*}-\zeta_{1}^{*}\right) k^{2}\right] \theta_{2 k_{0}}+\left[2 \zeta^{*}+\frac{5}{4}\left(\mu^{*}-\zeta_{2}^{*}\right) k^{2}\right] \rho_{2 k_{0}} } \\
+\frac{2}{3} i k \omega_{x 2 k_{0}}-\frac{1}{6} \eta^{*}(\alpha) k_{0}^{2} \omega_{0}^{2} e^{2 s_{\perp} \tau}=0
\end{aligned}
$$

Of course, the equations for $\rho_{2 k_{0}}$ and $\boldsymbol{\omega}_{2 k_{0}}$ remain the same as in the linear approximation. The results following from these approximated equations are compared with the numerical data from the DSMC method in Fig. 4. The system parameters are the same as in Figs. 2 and 3. The components of the velocity field are not plotted, the reason being that $\omega_{y 2 k_{0}}$ and $\omega_{z 2 k_{0}}$ are very small as compared with $\omega_{y k_{0}}$ and $\omega_{z k_{0}}$, and the longitudinal component remains of the order of the noise level in the time interval considered. For the relevant fields, i.e. density and temperature, the agreement is again quite good up to times larger than $\tau=100$. A comparison of the results in Figs. 3 and 4 indicates that for these times the contribution to the density field of the second Fourier component is much larger that the contribution from the first component. This leads us to conclude that the mechanism responsible for the development of density inhomogeneities and eventually density clusters is the nonlinear coupling of 

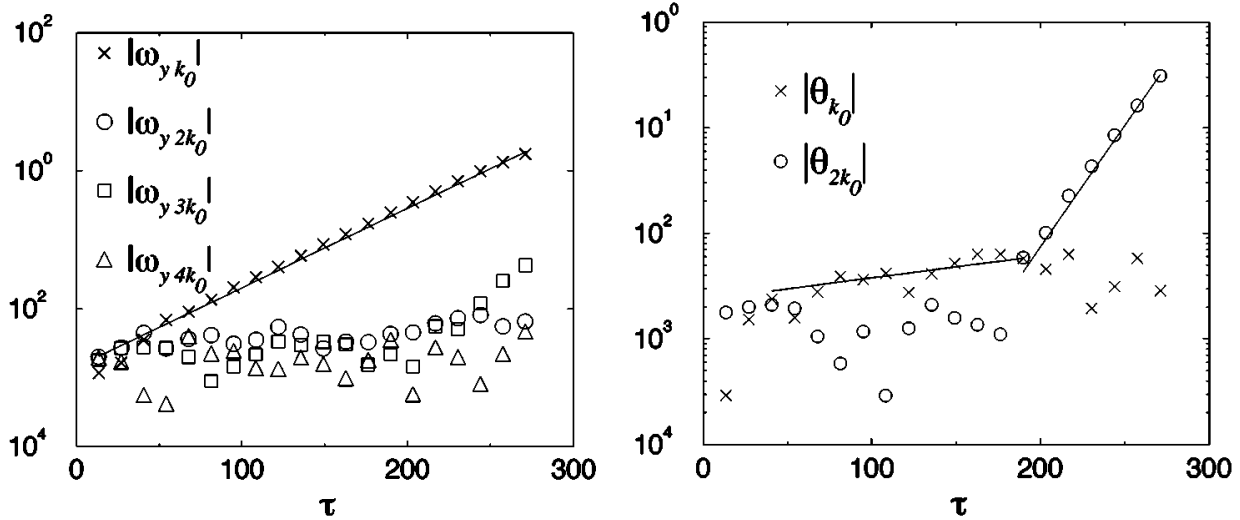

FIG. 5. Time evolution of the first few Fourier components of the spontaneous fluctuations of the hydrodynamic fields in a freely evolving system that is initially homogeneous. The parameters of the system are the same as in the previous figures. The straight lines have the slope predicted by the simple model in the main text, and have been located to obtain a good agreement with the numerical results.

the transversal components of the velocity field. After a short transient time, this effect dominates the growth of the density fluctuations.

It can be wondered to what extent the above conclusion, that has been reached by studying the response of the system to a given external perturbation, also applies to the spontaneous fluctuations taking place in a granular system. In Fig. 5 we present the results obtained by simulating the Boltzmann equation for a freely evolving system that is initially homogeneous. No external perturbation was introduced at any moment. Also in this case it is $\alpha=0.95$ and $L=60 \lambda$. We stress that again only gradients of the hydrodynamic fields in the $x$ direction are allowed, due to the simulated geometry. It is observed that the system spontaneously develops inhomogeneities in the hydrodynamic fields quite early. After an initial transient period, the growth of the Fourier components of the perturbations of the fields is approximately exponential on the time scale $\tau$. In addition, while the transversal velocity component is dominated by the first Fourier component over the time interval considered, the second Fourier components of the density and the temperature grow much faster than the first ones and, eventually, give the dominant contributions to the hydrodynamic fields. This agrees with the picture discussed above. A more quantitative comparison can be only made for the long time behavior of the fields, since it is not possible to fix a time in which the spontaneous perturbation of the freely evolving system takes place. The solid lines in Fig. 5 are the exponential laws obtained from our model equations in the long time limit, i.e., $\left|\omega_{y k_{0}}\right|$ $\sim \exp \left(s_{\perp} \tau\right),\left|\theta_{k_{0}}\right|,\left|\rho_{k_{0}}\right|,\left|\omega_{x k_{0}}\right| \sim \exp \left(s_{\|} \tau\right)$, and $\left|\theta_{2 k_{0}}\right|,\left|\rho_{2 k_{0}}\right|$, $\left|\omega_{x 2 k_{0}}\right| \sim \exp \left(2 s_{\perp} \tau\right)$. The positions of the drawn straight lines have been chosen such that roughly the best fit is obtained with the linear part of the curves. We believe that the agreement can be qualified as very good, taking into account the spontaneous character of the excitations we are describing. Figures 4 and 5 both indicate that the second Fourier components of the density field deviate from the exponential behavior for large times. That means that, as expected, our model equation is not valid for the final stages of the development of the instability. Even more, the figures suggest that the density perturbation is saturating to some finite value. More will be said about this point in the next section.

\section{DISCUSSION}

We have presented substantial qualitative and also quantitative evidence that, in the case of an inelastic low density gas, the leading mechanism responsible for the initial development of density inhomogeneities in the system is the nonlinear coupling effects coming from the transversal component of the flow field, and not the linear instability associated with the (linear) coupling between the longitudinal velocity fluctuations and the density field.

The results presented in the previous sections refer to the initial setup of inhomogeneities in the system. In fact, the largest inhomogeneity in density as observed for instance in Fig. 2, is about $20 \%$ of the average density. Therefore, a question arises in a natural way: what happens at later times? When the simulations are run longer, what is observed in all cases is that the density saturates to a steady profile, which does not depend at all on the initial conditions, i.e. on 


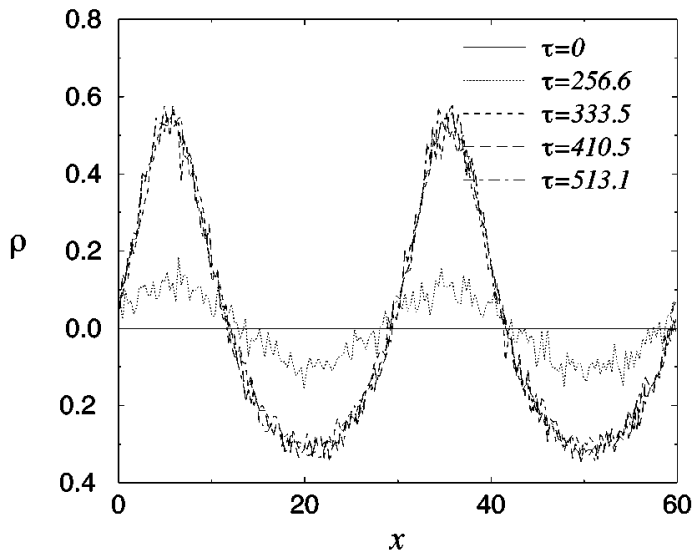

FIG. 6. Time evolution of the density profile along the $x$ direction for the same system as in Fig. 2. The initial condition was homogeneous and the system evolved freely. The same steady profile was reached starting from different initial conditions. The density is measured in the reduced dimensionless units defined in the text, and $x$ in units of the mean free path $\lambda$.

whether the system was or was not perturbed initially. As already mentioned above, there is some indication of this in Figs. 4 and 5. Figure 6 shows the time evolution of the density profile for the same system we have been considering throughout this paper. It is seen that it reaches a steady shape in which the ratio of the highest density to the lowest one is about 2. Similar results have been obtained for other values of the coefficient of restitution and of the system size. The main difference is qualitative. The inhomogeneity becomes stronger as the system size and the inelasticity increase, but it saturates in all cases. Since the Boltzmann equation does not contain any excluded volume effects, this saturation cannot be geometrical. Moreover, the smooth character of the steady density profile, and the relatively low inhomogeneity of the system suggest that this is a purely hydrodynamic effect.

We have also analyzed the linear or nonlinear character of the steady profile. In Fig. 7 the complete time evolution of the first few density modes is presented. This corresponds to the freely evolving case, so that the steady profile is spontaneously reached in this case. The system parameters are the same as in the previous figures. All the odd modes stay very small, while the even ones saturate to roughly equidistant values on the logarithm scale. In particular, the first mode does not increase in time, contrary to the linear result. Even more, if it is externally excited or suffers a spontaneous fluctuation, it always eventually decays below the noise level, indicating that it is asymptotically stable. We want to insist that these results are quite robust, and do not depend on the initial condition. Moreover, similar conclusions are reached from the analysis of systems in which gradients along several directions are allowed, although the situation becomes more complex since wave vectors with several components must be considered.

Since the density reached by the system remains in all regions of the same order of magnitude as its average value, it seems plausible to assume that the time evolution of the system is accurately described by the Boltzmann equation if the initial density is low enough. The situation may be different for very large systems with low coefficient of normal

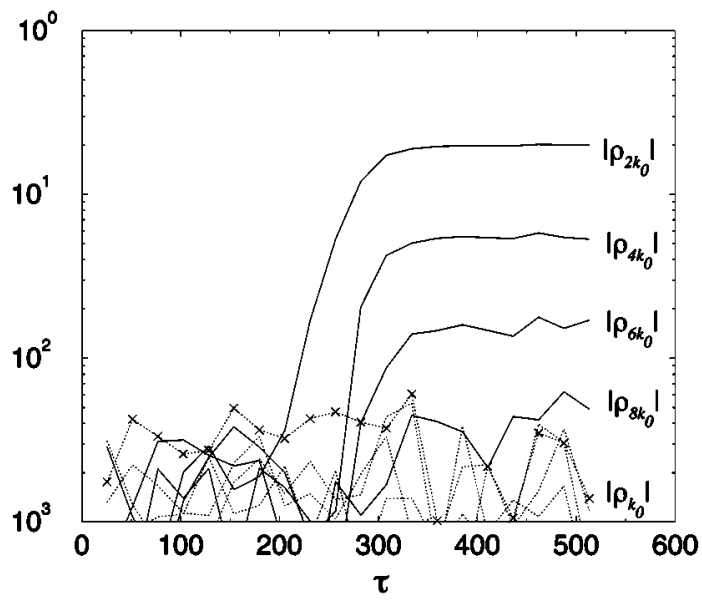

FIG. 7. Time evolution of the Fourier density modes for the same situation as in Fig. 6. Dotted lines correspond to the odd Fourier modes. Only the first one $\mid \rho_{k_{0}}$ has been labeled. Density and time are measured in the dimensionless units defined in the text.

restitution. If the system develops very high density regions, several orders of magnitude larger than the reference one, the Boltzmann equation is no longer valid to describe its time evolution. In any case, it seems clear that the inelastic Boltzmann equation does not lead to any singularity in real space, although the clustering as observed from the Boltzmann equation has all the characteristic features of the cluster instability in molecular dynamic simulations of inelastic hard particles, at least in the early stages [9]. Whether in a system of hard spheres or disks the density in the clustering is limited only by the densest possible fraction or if there is, in general, also a stationary density distribution is not clear for us presently. Nevertheless, we want to mention that some indication of the existence of a steady inhomogeneous profile for dense gases can be found in Ref. [3] (see, for instance, Fig. 9 there). The results we have presented suggest that for large systems and large inelasticity there is an inhomogeneous state, in which all the time dependence occurs through the temperature and which is stable, while the homogeneous cooling state is unstable. We are presentely working on this point, and results will be published elsewhere.

Finally, to put the results presented here in a proper context, it is worth mentioning that very recently a careful comparison of molecular dynamics and DSMC applied to granular media has been carried out [14]. The conclusion was that, except for large densities, large systems, and strong dissipation, both simulation techniques lead to similar results.

\section{ACKNOWLEDGMENTS}

It is a pleasure to thank Professor Frenkel for correspondence and also for sending us very clarifying unpublished work. This research was partially supported by Grant No. PB95-0534 from the Dirección General de Investigación Científica y Técnica (Spain).

\section{APPENDIX}

In this appendix, the expressions for the transport coefficients and source terms used in Eqs. (1)-(6) are given. The reduced shear viscosity, $\eta^{*}$ is 


$$
\eta^{*}(\alpha)=\left[\nu_{\eta}^{*}-\frac{1}{2} \zeta^{*}\right]^{-1}
$$

the reduced heat conductivity

$$
\kappa^{*}(\alpha)=\frac{2}{3} \frac{1+c^{*}}{\nu_{k}^{*}-2 \zeta^{*}},
$$

and the new reduced transport coefficient $\mu^{*}$

$$
\mu^{*}(\alpha)=2 \zeta^{*}\left(\kappa^{*}+\frac{c^{*}}{3 \zeta^{*}}\right)\left(2 \nu_{k}^{*}-3 \zeta^{*}\right)^{-1} .
$$

The coefficients $\zeta^{*}, \zeta_{1}^{*}$, and $\zeta_{2}^{*}$, appearing in the source of the energy term, are

$$
\begin{gathered}
\zeta^{*}=\frac{5}{12}\left(1-\alpha^{2}\right)\left(1+\frac{3 c^{*}}{32}\right), \\
\zeta_{1}^{*}=\frac{1}{16}\left(1-\alpha^{2}\right) \kappa^{*}\left(1-\frac{77 c^{*}}{64}\right)\left[\nu_{\zeta}^{*}-\frac{5}{2} \zeta^{*}-\frac{5}{32}\left(1-\alpha^{2}\right)\right. \\
\left.\times\left(1+\frac{35 c^{*}}{64}\right)\right]^{-1},
\end{gathered}
$$

$$
\begin{aligned}
\zeta_{2}^{*}= & {\left[\zeta_{1}^{*} \zeta^{*}+\frac{1}{16}\left(1-\alpha^{2}\right) \mu^{*}\left(1-\frac{77 c^{*}}{64}\right)\right] } \\
& \times\left[\nu_{\zeta}^{*}-2 \zeta^{*}-\frac{5}{32}\left(1-\alpha^{2}\right)\left(1+\frac{35 c^{*}}{64}\right)\right]^{-1} .
\end{aligned}
$$

In these expressions, $c^{*}, \nu_{\eta}^{*}, \nu_{k}^{*}$, and $\nu_{\zeta}^{*}$ are functions of $\alpha$ given by

$$
\begin{gathered}
c^{*}=\frac{32(1-\alpha)\left(1-2 \alpha^{2}\right)}{81-17 \alpha+30 \alpha^{2}(1-\alpha)}, \\
\nu_{\eta}^{*}=\left[1-\frac{1}{4}(1-\alpha)^{2}\right]\left[1-\frac{1}{64} c^{*}\right], \\
\nu_{k}^{*}=\frac{1}{3}(1+\alpha)\left[1+\frac{33}{16}(1-\alpha)+\frac{19-3 \alpha}{1024} c^{*}\right], \\
\nu_{\zeta}^{*}=\frac{1+\alpha}{192}\left[271-207 \alpha+30 \alpha^{2}-30 \alpha^{3}\right. \\
\left.+\frac{c^{*}}{64}\left(137-9 \alpha-30 \alpha^{2}+30 \alpha^{3}\right)\right] .
\end{gathered}
$$

[1] I. Goldhirsch and G. Zanetti, Phys. Rev. Lett. 70, 1619 (1993); I. Goldhirsch, M.L. Tan, and G. Zanetti, J. Sci. Comput. 8, 1 (1993).

[2] S. McNamara and W.R. Young, Phys. Rev. E 50, R28 (1994).

[3] S. McNamara and W.R. Young, Phys. Rev. E 53, 5089 (1996).

[4] P. Deltour and J.L. Barrat, J. Phys. I 7, 137 (1997).

[5] R. Brito and M. H. Ernst, Europhys. Lett. 43, 497 (1998).

[6] C. S. Campbell, Annu. Rev. Fluid Mech. 22, 57 (1990).

[7] J. J. Brey, J. W. Dufty, C. S. Kim, and A. Santos, Phys. Rev. E 58, 4638 (1998).

[8] J. J. Brey, M. J. Ruiz-Montero, and D. Cubero, Phys. Rev. E
54, 3664 (1996).

[9] J. J. Brey, M. J. Ruiz-Montero, and F. Moreno, Phys. Fluids 10, 2976 (1998); J. J. Brey and M. J. Ruiz-Montero (unpublished).

[10] G. Bird, Molecular Gas Dynamics and the Direct Simulation of Gas Flows (Clarendon Press, Oxford, 1994).

[11] A. Goldshtein and M. Shapiro, J. Fluid Mech. 282, 75 (1995).

[12] J. J. Brey, F. Moreno, and M. J. Ruiz-Montero, Phys. Fluids 10, 2965 (1998).

[13] J. J. Brey, M. J. Ruiz-Montero, and D. Cubero (unpublished). [14] S. Luding, M. Muller, and S. McNamara (unpublished). 